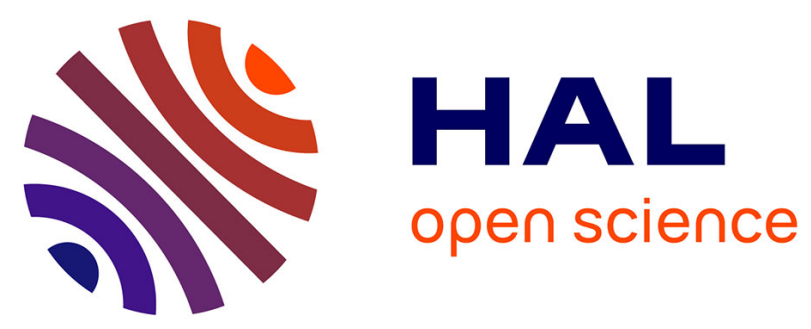

\title{
ON THE ROLE OF QUANTUM AND STATISTICAL EFFECTS IN THE LIQUID GAS PHASE TRANSITION OF HOT NUCLEI
}

\author{
A. Blin, B. Hiller, H. Reinhardt, P. Schuck
}

\section{- To cite this version:}

A. Blin, B. Hiller, H. Reinhardt, P. Schuck. ON THE ROLE OF QUANTUM AND STATISTICAL EFFECTS IN THE LIQUID GAS PHASE TRANSITION OF HOT NUCLEI. International Conference on Heavy Ion Nuclear Collisions in the Fermi Energy Domain, Hicofed 86, 1986, Caen, France. pp.C4-423-C4-426, 10.1051/jphyscol:1986450 . jpa-00225816

\section{HAL Id: jpa-00225816 https://hal.science/jpa-00225816}

Submitted on 1 Jan 1986

HAL is a multi-disciplinary open access archive for the deposit and dissemination of scientific research documents, whether they are published or not. The documents may come from teaching and research institutions in France or abroad, or from public or private research centers.
L'archive ouverte pluridisciplinaire HAL, est destinée au dépôt et à la diffusion de documents scientifiques de niveau recherche, publiés ou non, émanant des établissements d'enseignement et de recherche français ou étrangers, des laboratoires publics ou privés. 


\title{
ON THE ROLE OF QUANTUM AND STATISTICAL EFFECTS IN THE LIQUID GAS PHASE TRANSITION OF HOT NUCLEI
}

\author{
A.H. BLIN, B. HILLER, H. REINHARDT* and P. SCHUCK* * \\ Institut für Theoretische Physik, Universität Regensburg. \\ D-8400 Regensburg, F.R.G. \\ "Zentralinstitut für Kernforschung, Rossendorf, Postfach 19. \\ D-8051 Dresden, D.R.G. \\ * ISN, 53, Avenue des Martyrs, F-38026 Grenoble Cedex, France
}

Résumé - La transition de phase Liquide-gaz dans la matière nucléaire chaude due aux fluctuations quantiques et statistiques est étudieé dans un modèle microscopique de la nucléation. Ce modèle est une version hydrodynamique de la théorie de champs moyen dépendant du temps imaginaire aux températures finies.

Abstract - The triggering of the Liquid-gas phase transition in hot nuclear matter by quantum and statistical fluctuations is studied in a microscopic approach to nucleation, which is a fluid-dynamical version of the imaginary time dependent mean field theory at finite temperature.

\section{I - INTRODUCTION}

The equation of state of hot nuclear matter exhibits a typical van der. Waals behaviour and indicates the coexistence of a liquid phase at normal nuclear matter density and of a gas phase (at lower densities) which consists of a free gas of nucleons, deuterons, etc. 1-3). At low temperatures, where statistical fluctuations can be discarded in a classical theory, the two homogeneous phases which correspond to the liquid and gas are stable and may have different or equal energy densities. However, in a quantum theory the state of higher energy is rendered unstable through barrier penetration and a phase transition may take place. Phase transitions are also possible via thermodynamical fluctuations. A new phase may be formed in small volumes, say, bubble in a nuclear matter environment, which are stable if they have a certain size, being energetically favourable in the interplay of volume and surface energies.

The role of quantum and statistical fluctuations has been first analyzed in a microscopic description in the recent work of reference ${ }^{4)}$. There a relativistic model field theory of nuclear systems (Walecka's model 5), has been used to study the liquid vapour phase transitions applying euclidean space path integral techniques 6). In the present work we use a similar dynamical treatment to study phase transitions in a non-relativistic description of the nuclear many-body system with effective skyrme forces. The quantity which describes the decay of one of the metastable phases into the stable one ("nucleation rate") has a.W.K.B. Like form

$$
W=B \exp \left(-\tilde{A}_{i}\right)
$$

where $\tilde{A}_{j}$ is the minimized classical (euclidean) action associated with bubble or droplet formation and $B$ is a factor which arises from the functional integral over the fluctuations. Equation (I) is applicable to bubble formation (or nucleation) triggered by quantum as well as statistical fluctuations but the corresponding action differs in the two cases. By minimizing the action one obtains an equation of motion for the bubble or droplet radius as function of the imaginary time. This equation has several solutions. Two of them are trivial static solutions and correspond to the homogeneous gas and liquid phase respectively. One is static but non trivial, representing a bubble (droplet) on the background of a constant liquid (gas) phase and arises due to statistical fluctuations. The action corresponding to this solution 
enters in the calculation of statistical fluctuations. Finally there exists an (imaginary) time dependent solution, which describes the formation of bubbles (droplets) through barrier penetration or quantum fluctuations.

\section{II - THE ACTION PRINCIPLE FOR FINITE TEMPERATURES}

At finite temperatures, the euclidean action, defined as $(-i)$ times the continuation of the real time section to imaginary times $\tau$ is given in the fluid dynamical approximation by $B / 2$

$$
A[\rho]=\int_{-\beta / 2}^{\beta / 2} d \tau\left(T_{n}(\dot{\rho} x)-\frac{m}{2} T_{\Omega}\left(\rho u^{2}\right)-\Omega[\rho]\right)
$$

with the boundary condition $\rho(B / 2)=\rho(-\beta / 2)$ and the inverse temperature $T$ of the system. Here $\rho$ is the time even part of the density matrix, the field $X$ is assumed,to be local in space, and the velocity field $u$ is associated to the field $X$ by $\vec{u}=\frac{1}{m} \vec{\nabla} X$. The quantity $\Omega$ in eq. (II.1) is the thermodynamic potential, a functional of $p$

$$
\Omega[\rho]=E[\rho]-\mu T_{\Omega}(\rho)-S / \beta
$$

where $\mu$ is the chemical potential, $S$ the entropy and E[o] the mean field energy functional.

The variations of the action integral with respect to $\chi$ and $p$ lead to the fluid dynamics equations. Since eq. (II.1) is subject to the boundary condition $\rho(B / 2)=$ $=\rho(-\beta / 2)$, the solution is not as usual an initial value problem, but rather a boundary value problem with periodic conditions. This renders the problem quite difficult to handle.

\section{III - SIMPLIFICATION OF THE MODEL}

To get a first insight into the non trivial mean-field solutions describing the nucleation process (bubble or droplet formation) we resort to several approximations which we list as follows:

(i) we assume radial symmetry (for the bubble or droplet)

(ii) the density of a bubble solution is parametrized as

$$
\rho=\rho_{L}+\left(\rho_{c}-\rho_{L}\right) /\{1+\exp [(r-R(c)) / a]\}
$$

and the density of a droplet solution is obtained by interchaning the roles of $\rho_{G}$ and $\rho_{L}$.

Here $P_{G}$ and $\rho_{L}$ are the values of the densities which result from a Maxwell construction in a nuclear matter calculation. Then the coexistence of the gas and liquid phases is characterized by densities of equal chemical potential $\mu$

$\mu\left(\rho_{G}\right)=\mu\left(\rho_{L}\right)$.

The imaginary time $\tau$ enters through the parameter $R$. The parameter $a$, which represents the surface thickness of the droplet, is kept constant for a fixed temperature. (iji) The mean field hamiltonian is calculated in the finite temperature Thomas Fermi approximation with a Skyrme interaction 3 ).

(iv) We neglect Coulomb effects and assume symmetric nuclear matter.

The choice of parametrization ( $i$ ) corresponds to our belief that hot compressed nucLear matter may desintegrate to give rise to bubbles. at much lower than saturation densities through statistical and quantum fluctuations. In a similar fashion we also consider the possibilities that a hot nucleon gas may materialize in small droplets. This picture is substantiated by the equation of state of hot nuclear matter, which exhibits a typical Van der Waals behaviour.

In heavy ion reactions the process of bubble formation is probably more relevant than the droplet formation. Droplet formation could in principle be important during the strong expansion phase of a high energy nuclear reaction. However, we will see below that this occurs in our model only for densities much lower than the usually accepted value of the freeze - out density.

\section{IV - THE VELOCITY FIELD AND THE MASS PARAMETERS}

The velocity field $\vec{u}$ which appears in the action integral is obtained using the conti- 
nuity equation (spherical coordinates, radial symmetry) which can be integrated to yield

$$
u(r)=-\frac{1}{r^{2}} \int_{0}^{r} d r^{\prime} r^{2} \dot{\rho} / \rho
$$

This equation allows to determine the velocity field which corresponds to a certain density. To calculate the action integral (II.1) using the parametrization (III.1), it is necessary to know how $\rho$ evolves in the imaginary time $\tau$. Since in (III.1) $\rho$ depends on $\tau$ only through $R(T)$, we vary the action $A$ with respect to $R$ and obtain the following equation of motion for $R$

$$
\frac{d \Omega}{d R}-\frac{d \alpha}{d R} \dot{R}^{2}-2 \alpha \ddot{R}=0
$$

where $\alpha$ figures as mass parameter.

\section{$\checkmark$ - THE THERMODYNAMICAL POTENTIAL $\Omega$}

The thermodynamical potential corresponding to bubble formation is defined as

$$
\widetilde{\Omega}_{B}[\rho]=\Omega[\rho]-\Omega\left[\rho_{L}\right] \text {. }
$$

Here $\Omega\left[\rho_{L}\right]$ is the thermodynamic potential associated with the Liquid background of eq. (II.I.1). In Fig. 1, we depict $\widetilde{\Omega}_{B}$ for $T=9 \mathrm{MeV}$. Since $\rho \rightarrow \rho_{\mathrm{L}}$ as $R \rightarrow-\infty$ one approaches from the left the potential minimum corresponding to the liquid phase, which is substracted off in the calculation of $\widetilde{\Omega}_{B}$. Therefore $\widetilde{\Omega}_{B}$ vanishes for $R \rightarrow-\infty$. For large values of $R, \rho \rightarrow \rho_{G}$, and one approaches from the right the potential minimum of the gas phase. The barrier between the two phases is due to the surface energy. After reaching the saturation value, the volume energy of the bubble starts to dominate over the surface energy, which explains the fall off of $\zeta_{B}$ after a certain critical R.

\section{VI - QUANTUM FLUCTUATIONS}

It is necessary to solve explicitly the imaginary time equation of motion for $R$, eq. (IV.2) with the appropriate boundary condition. The solution of eq. (IV.2) shows how the radius $R$ of the droplet or bubble behaves in the course of the imaginary time $\tau$. Figure 2 shows the imaginary time evolution of a bubble radius $R(\tau)$ for $\mu=-20.5$ MeV at $\mathrm{T}=0.5 \mathrm{MeV}$.

In Table 1 we show the initial values of $R$ which are compatible with quantum fluctuations at several values of the chemical potantial $\mu$, and the resulting bubble formation probabilities at the temperature $\mathrm{T}=0.5 \mathrm{MeV}$.

\begin{tabular}{|c|c|c|c|}
$R_{i}(\mathrm{fm})$ & $R_{c r}$ & $\mu$ & $W_{\text {quant } / \mathrm{B}}$ \\
\hline-0.5 & 2.1 & -20.5 & $6 \times 10^{-6}$ \\
0 & 1.5 & -20.7 & $8.5 \times 10^{-3}$ \\
\hline
\end{tabular}

\section{VII - STATISTICAL FLUCTUATIONS}

Statistical fluctuations correspond to the non trivial static solutions of eq. (IV.2) that is $(\dot{R}=0, \vec{R}=0)$ and $d \Omega / d R=0$. Therefore the non trivial solution is $\widetilde{\Omega}_{j}(R)=$ $=\widetilde{\Omega}_{i}\left(R_{c r}\right)$ where $R_{c r}$ is the critical radius for which $\widetilde{\Omega}_{i}(R)$ acquires its maximum. Fig. 3 show the resulting critical bubble radii and nucleation rates at $T=9 \mathrm{MeV}$ as function of the density $R$ :

REFERENCES - 1) 6. Sauer, H. Chandra and U. Mosel, Nucl. Phys. A 264 (1976) 221;

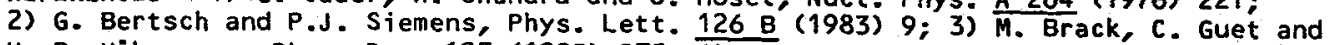
H.-B. Mákansson, Phys. Rep. 123 (1985) 275; 4) H. Reinhardt and H. Schulz, Nucl. Phys. A 432 (1985) 630; 5) J.D. Walecka, Ann. Phys. 83 (1974) 491; Phys. Lett. 59 B (1975) 109; 6) S. Coleman, Phys. Rev. D 15 (1977) 2929; H. Reinhardt, Nucl. Phys. A 367 (1981) 269. 


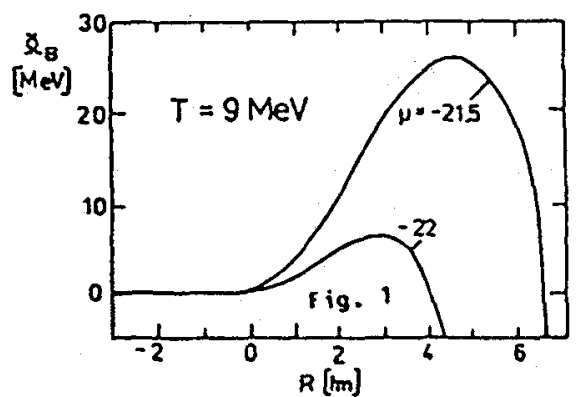

Fig. 1 - The thermodynamical potential $\tilde{\Omega}$, of a bubble on a 1iquid background as function of the radius $R$ of the bubble for the isotherm $T=9 \mathrm{MeV}$ and two values of the chemical potential (in MeV).

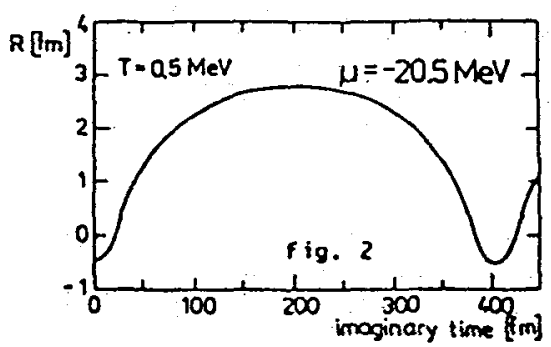

Fig. 2 - Time-dependent solution to the equation of motion IV.2. The bubble radius $R$ is shown as function of the imaginary time $\tau$, corresponding to the solution inside the inverted thermodynamical potential for $\mu=-20.7 \mathrm{MeV}$ and $\mathrm{T}=0.5 \mathrm{MeV}$. The minimum of $R$ lies on one turning point of the potential.

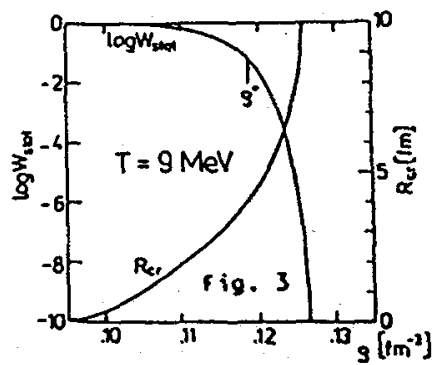

Fig. 3 - The Togarithms of the normalized transition probabilities Wstat per unit time and volume for bubble formation (left scale) due to statistical fluctuations, at $T=9 \mathrm{MeV}$ and shown as function of $\mathrm{O}_{\mathrm{L}}$. Right scale : critical bubble radius $\mathrm{R}_{\mathrm{cr}}$. 